Pacific Journal of Mathematics

PSEUDOCOMPACT AND STONE-WEIERSTRASS PRODUCT 


\title{
PSEUDOCOMPACT AND STONE-WEIERSTRASS PRODUCT SPACES
}

\author{
R. M. Stephenson, JR.
}

In $1960 \mathrm{H}$. Tamano proved that for pseudocompact completely regular spaces $X$ and $Y$, (i) $X \times Y$ is pseudocompact if and only if $p r_{X}$ is $z$-closed, and (ii) $X \times Y$ is pseudocompact if one of $X$ and $Y$ is a $k$-space.

In 1979 C. E. Aull asked if every product of functionally regular $\mathrm{SW}$ spaces is an $\mathrm{SW}$ space, and he proved that for a family of functionally regular $\mathrm{SW}$ spaces, (iii) their product is an SW space if and only if it is pseudocompact.

The main results of this paper will answer Aull's question affirmatively and prove that (i), (ii), and (iii) hold for strongly functionally Hausdorff spaces.

A topological space $X$ is said to be functionally Hausdorff if $C(X)$ (or $C^{*}(X)$ ), the set of (bounded) continuous real valued functions defined on $X$, is point separating; given a functionally Hausdorff space $X, w X$ will denote the completely regular space which has the same points and continuous real valued functions as those of $X$. A functionally Hausdorff space $X$ will be called an SW space if every point separating subalgebra of $C^{*}(X)$ which contains the constants is uniformly dense in $C^{*}(X)$ (or, equivalently, if $w X$ is compact). A Hausdorff space $X$ will be called functionally regular (strongly functionally Hausdorff) if for each point $p \in X$ and neighborhood $V$ of $p$ (such that $V=\mathrm{Cl}(\operatorname{Int}(V))$ ) there is a zero set $Z$ of $X$ with $p \in Z \subset V$.

In addition to the above, a proof will be given that every feebly compact product of SW spaces is an SW space (which will partially answer another question of Aull), and an example will be given of a non SW product space each of whose finite subproducts is an SW strongly functionally Hausdorff space. This example will show that there exists a sequence $\left\{X_{n}\right\}$ of strongly functionally Hausdorff spaces whose product $X=\Pi\left\{X_{n}\right\}$ and whose finite subproducts $X_{F}=$ $\Pi\left\{X_{n}: n \in F\right\}, F$ finite, have the following curious properties: each $w X_{F}=\Pi\left\{w X_{n}: n \in F\right\}$ and is compact, but $w X$ fails to be pseudocompact and thus does not equal $\Pi\left\{w X_{n}\right\}$.

We will obtain several of these results by proving the following for a product space $X=\Pi\left\{X_{a}\right\}$, where each $X_{a}$ is a pseudocompact strongly functionally Hausdorff space: If $X$ is pseudocompact, or if each factor space $X_{a}$ is functionally regular, then $w X=$ $\Pi\left\{w X_{a}\right\}$. 
2. Some recent results. By a $P$-closed space, where $P$ is some property of topologies, one just means a $P$-space $X$ such that $X$ is a closed subspace of every $P$-space in which it can be embedded.

Now we can state a characterization theorem.

THEOREM 1. Let $X$ be a functionally Hausdorff space. The following are equivalent.

(i) $X$ is functionally Hausdorff-closed.

(ii) $w X$ is compact.

(iii) $X$ is an SW space.

In $1947 \mathrm{E}$. Hewitt proved that every completely regular SW space is compact [13], and in 1957 B. Banaschewski used Hewitt's result to establish the equivalence of (ii), (iii), and a filter condition [4]. The equivalence of (i) with (ii) was obtained by me [16] several years later.

In 1966 we began trying to determine if the property SW is productive but succeeded only in showing that the product of SW spaces is an SW space if all but one of the factors are compact. Two results obtained later are the following.

THEOREM 2. [18] Let $X$ and $Y$ be SW spaces. The following are equivalent.

(i) $X \times Y$ is an SW space.

(ii) $p r_{X}: X \times Y \rightarrow X$ is z-closed, i.e., for every zero set $Z$ of $X \times Y, p r_{X}(Z)$ is a closed subset of $X$.

(iii) $w(X \times Y)=w X \times w Y$.

THEOREM 3. [19] Let $X=\Pi\left\{X_{a}: a \in A\right\}$, where each $X_{a}$ is an SW space. Then the following are equivalent.

(i) $X$ is an SW space.

(ii) For any finite set $B \subset A, p r_{B}: X \rightarrow \Pi\left\{X_{b}: b \in B\right\}$ is z-closed.

(iii) $w X=\Pi\left\{w X_{a}: a \in A\right\}$.

One interesting consequence of Theorem 2 is that if $X$ and $Y$ are SW spaces, one of which is a $k$-space, then $X \times Y$ is an SW space [18]. Thus, every finite product of first countable SW space is an SW space. So far as $I$ know, the following question has not been answered.

Question 4. [19] Is every product of first countable SW spaces an $\mathrm{SW}$ space?

Recently, Aull discovered that there is a close relationship between pseudocompact and SW product spaces, at least for a large 
family of spaces, namely, van Est's functionally regular spaces [22]. (It might be mentioned here that included among functionally regular spaces are, besides completely regular spaces, all spaces and products of spaces in which points are zero sets; e.g., see [1].)

THEOREM 5. [2] A product of functionally regular SW spaces is an SW space if and only if it is pseudocompact.

In view of Theorem 5 , it is natural to ask the following.

Question 6. [2] (i) Is every pseudocompact product of SW spaces an SW space, and (ii) is every product of functionally regular SW spacss an SW space?

3. Some new results, Partial answers to Questions 4 and 6 will be given, and we will obtain the results listed in the introduction.

A topological space is called feebly compact [15] (or lightly compact [3]) if every locally finite system of open sets is finite. It is well known that feeble compactness implies pseudocompactness, and in completely regular spaces the two concepts are equivalent.

LEMmA 7. Let $X$ and $Y$ be topological spaces, and suppose that $X \times Y$ is feebly compact. Then $p r_{X}: X \times Y \rightarrow X$ is z-closed.

In [21] Tamano proved this (and more) for completely regular spaces. The short proof in [8] of the "only if" part of Tamano's theorem can be used here without modification to establish Lemma 7.

Theorem 8. Let $X=\Pi\left\{X_{a}: a \in A\right\}$, where each $X_{a}$ is an SW space, and suppose that $X$ is feebly compact. Then $X$ is also an SW space.

Proof. For any subset $B$ of $A, p r_{B}: X \rightarrow \Pi\left\{X_{b}: b \in B\right)$ is z-closed by Lemma 7 , and so by Theorem $3, X$ is an SW space.

Since it is known that every product of first countable feebly compact spaces is feebly compact [15], Theorem 8 improves the result obtained in [19] that every product of first countable feebly compact SW spaces is an SW space.

It might also be mentioned that since feeble compactness of a product is determined by feeble compactness of countable subproducts, one can prove that $X$ satisfies the hypothesis of Theorem 8 by just proving that each countable subproduct of $X$ satisfies it. 
Lemma 9. Let $X$ be a pseudocompact Hausdorff space, and suppose that $P \subset C(X)$ is such that for each point $x \in X$ and neighborhood $V$ of $x$ in $X$ there exists $f \in P$ with $f(x) \notin f(X \backslash \bar{V})$. Let $p X$ denote the completely regular space whose points are those of $X$ and whose topology is the weak topology determined by $P$. Then $p X=$ $w X$.

Proof. Since $P \subset C(X)$, every open set of $p X$ is open in $w X$. To show that each open subset of $w X$ is open in $p X$, it suffices to prove that every zero set of $X$ is a closed subset of $p X$.

Let $Z$ be a zero set of $X$. Consider an arbitrary point $x$ in $X \backslash Z$. We will prove that there is a neighborhood $W_{x}$ of $x$ in $p X$ with $W_{x} \cap Z=\phi$.

There exists a neighborhood $V$ of $x$ in $X$ with $\bar{V} \cap Z=\phi$. By hypothesis one can find a function $f \in P$ with $f(x) \notin f(X \backslash \bar{V})$. Because $X$ is pseudocompact and $f \in C(X)$ (since $P \subset C(X)$ ), it follows from an argument in [2] (see also [14]) that $f(Z)$ is a closed subset of $f(X)$. Thus there is a continuous mapping $h$ of $f(X)$ into the unit interval which vanishes at $f(x)$ and equals 1 on $f(Z)$. The mapping $i=h \circ f$ is in $C(p X)$, and so $W_{x}=i^{-1}([0,1))$ is an open neighborhood of $x$ in $p X$ with $W_{x} \cap Z=\phi$.

It will be convenient to use the easily verified fact that a Hausdorff space $X$ is strongly functionally Hausdorff provided that for each point $x \in X$ and neighborhood $V$ of $x$ there exists $f \in C(X)$ with $f(x) \notin f(X \backslash \bar{V})$. We also note that strongly functionally Hausdorff is a topological and productive property which is slightly weaker than functionally regular, though equivalent with it in regular spaces. Its usefulness is illustrated below.

Lemma 10. Let $X=\Pi\left\{X_{a}: a \in A\right\}$, where each $X_{a}$ is strongly functionally Hausdorff, and suppose that $X$ is pseudocompact. Then $w X=\Pi\left\{w X_{a}: a \in A\right\}$.

Proof. The ring $P$ generated by $\left\{f \circ p r_{a}: a \in A\right.$ and $\left.f \in C\left(X_{a}\right)\right\}$ satisfies the hypothesis of Lemma 9 , so it follows that the space $p X=\Pi\left\{w X_{a}: a \in A\right\}$ is the same as $w X$.

Combining Lemma 10 and Theorem 3, one obtains the following extension of Aull's theorem [2].

THEOREM 11. A product of strongly functionally Hausdorff SW spaces is an SW space if and only if it is pseudocompact.

The next theorem strengthens an analogous result obtained by Tamano in [21] for completely regular spaces. 
THEOREM 12. Let $X$ and $Y$ be pseudocompact spaces.

(i) If $X$ and $Y$ are strongly functionally Hausdorff spaces, then $X \times Y$ is pseudocompact if and only if $p r_{X}$ is z-closed.

(ii) If $X$ is a Hausdorff $k$-space and $Y$ is a strongly functionally Hausdorff space, then $X \times Y$ is pseudocompact.

Proof. (i) According to [17, 4.8(i), due to the referee], if $X$ and $Y$ are pseudocompact spaces and $p r_{X}$ is $z$-closed, then $X \times Y$ is pseudocompact. Conversely, suppose $X \times Y$ is pseudocompact. Then the completely regular spaces $w X$ and $w Y$ have a pseudocompact product, and so by Tamano's theorem, the mapping $p r_{w X}: w X \times$ $w Y \rightarrow w X$ is $z$-closed. Since $w X \times w Y$ and $X \times Y$ have the same zero sets by Lemma 10 , it follows that $p r_{X}: X \times Y \rightarrow X$ is $z$-closed.

(ii) The proof needed here is similar to the corresponding one in [21]. Let $Z$ be a zero set of $X \times Y$, and consider any compact subset $K$ of $X$. The space $K \times Y$ is a pseudocompact product of strongly functionally Hausdorff spaces, so by Lemma 10, $K \times Y$ and $K \times w Y$ have the same zero sets. Thus $Z \cap(K \times Y)$ is a zero set of the feebly compact space $K \times w Y$, and hence by Lemma 7, the set $K \cap p r_{X}(Z)=p r_{K}(Z \cap(K \times Y))$ is a closed subset of $K$. Therefore, $p r_{X}(Z)$ is a closed subset of $X$, and $p r_{X}: X \times Y \rightarrow X$ is $z$-closed. By [17, 4.8(i)], $X \times Y$ must be pseudocompact.

In [20] it was shown that the property SW is not productive an example was constructed of a countably compact SW space $X$ such that $X \times X$ is not even pseudocompact. We will now show that there exists a strongly functionally Hausdorff product space $X$ such that each finite subproduct of $X$ is an SW space, but $X$ itself is not an SW space. In either of these two constructions, the space $X$ can also be used as an example to show that not every strongly functionally Hausdorff space is functionally regular.

EXAMPLE 13. Let $N$ denote the set of natural numbers, with the discrete topology, and let $G_{n}=N \cup T_{n}$, where $N \cap T_{n}=\phi$, be a subspace of $\beta N, n \in N$, such that $\cap\left\{T_{n}: n \in N\right\}=\phi$, but for each finite set $B \subset N$, the product space $\Pi\left\{G_{n}: n \in B\right\}$ is pseudocompact. By a construction of W. W. Comfort [7], such spaces exist.

Let $B_{n}$ be the space whose points are those of $\beta N$ and whose topology is the collection of all sets of the form $S \cup\left(W \cap\left(\beta N \backslash T_{n}\right)\right)$, where $S$ and $W$ are open subsets of $\beta N$. Let $I=\{1,2\}$ have the discrete topology, $P_{n}=B_{n} \times I$ have the product topology, and $Y_{n}=$ $G_{n} \times\{1\} \cup B_{n} \times\{2\}$ have the topology it inherits from $P_{n}$. Let $R_{n}$ be the equivalence relation on $Y_{n}$ defined by the rule $(v, i) R_{n}(w, j)$ if (i) $v=w$ and $i=j$, or (ii) $v=w \in T_{n}$. Denote the quotient space $Y_{n} / R_{n}$ by $X_{n}$. We will continue to use the symbols $(\mathrm{v}, \mathrm{i})$ for the 
points of $X_{n}$; thus, $(\mathrm{v}, 1)=(\mathrm{v}, 2)$ for $v \in T_{n}$.

The spaces $X_{n}, n \in N$, have the following properties.

(i) Each $X_{n}$ is a strongly functionally Hausdorff SW space.

(ii) Each $G_{n} \times\{1\}$ is feebly compact, and each $B_{n} \times\{2\}$ is a Hausdorff-closed space.

(iii) For each finite subset $B$ of $N, X_{B}=\Pi\left\{X_{n}: n \in B\right\}$ is a feebly compact and (hence) an SW space.

(iv) The space $X=\Pi\left\{X_{n}: n \in N\right\}$ is not pseudocompact.

(v) Each $X_{n}$ fails to be functionally regular.

Proof. (i) The proofs that each $X_{n}$ is functionally Hausdorff and SW are very similar to corresponding proofs given in [20].

To see that $X_{n}$ is strongly functionally Hausdorff, first note that each point of $G_{n} \times I$ has a neighborhood base consisting of clopen sets. Next, consider a point $(v, 2) \in X_{n}$, where $v \notin G_{n}$, and a basic open neighborhood of $(v, 2)$, say

$$
V=W \cap\left(\left(\beta N \backslash T_{n}\right) \times\{2\}\right) .
$$

It is easy to show that $\bar{V}=K \backslash(N \times\{1\})$, where $K$ is the clopen set $X_{n} \cap\left(\mathrm{Cl}_{\beta N} W\right) \times\{1,2\}$. Let $g, f_{k}, k \in N$, be the $\{0,1\}$ valued functions in $C\left(X_{n}\right)$ determined by the rules $g(x)=0$ if and only if $x \in K$, and $f_{k}(y)=1$ if and only if $y=(k, 1)$. Then $f=g+\sum 2^{-k} f_{k}$ is in $C\left(X_{n}\right)$ and satisfies $f(v, 2)=0 \notin f\left(X_{n} \backslash \bar{V}\right)$.

(ii) It is known (and not difficult to prove) that (1) every open filter base on a space such as $B_{n} \times\{2\}$ has an adherent point (e.g., see [6] or [5]), and (2) a Hausdorff space is Hausdorff-closed if and only if every open filter base on the space has an adherent point ([5], [6], [23, 17K]). In [7] Comfort proves that each of the completely regular spaces $G_{n}$ is pseudocompact. Thus $G_{n} \times\{1\}$ is feebly compact.

(iii) To prove that $X_{B}$ is an SW space, it suffices (by Theorem 8 or 11 ) to prove that $X_{B}$ is feebly compact. Since any finite union of feebly compact spaces is feebly compact, it is enough to note that $X_{B}$ is a union of $2^{|B|}$ spaces, each topologically having the form $A \times F$, where $A$ is a product of Hausdorff-closed spaces, and $F$ is a finite (hence feebly compact) subproduct of the space in [7]. For, the property Hausdorff-closed is productive [23, 17L], and given a Hausdorff-closed space $A$ and feebly compact space $F$, one can show that for a countable open filter base $\mathscr{\mathscr { C }}$ on $A \times F$ and adherent point $f$ of $p r_{F}(\mathscr{C})$, if $a \in A$ is any adherent point of the open filter base

$$
p r_{A}\left(\left\{U \cap \operatorname{pr}_{F}^{-1}(V): V \text { is open, } f \in V, U \in \mathscr{U}\right),\right.
$$

then $(a, f)$ is an adherent point of $\mathscr{C}$, i.e., $A \times F$ is feebly compact. 
(iv) The proof needed here is a modification of one in [7]. For each $n \in N$ let $U_{n}$ be the clopen set

$$
\{x \in X: x(i)=(n, 1), i \in N \text { and } i \leqq n\},
$$

and let $\mathscr{C}=\left\{U_{n}: n \in N\right\}$. Then, as in [7], because $\cap\left\{T_{n}: n \in N\right\}=\phi$, one can prove the family $\mathscr{C}$ is locally finite. For each $n \in N$ let $f_{n}$ be the characteristic function of $U_{n}$, and define $f=\sum n f_{n}$. By the local finiteness of $\mathscr{\mathscr { C }}, f \in C(X) \mid C^{*}(X)$.

(v) This is an immediate consequence of Lemma 22 and Remark 23.

REMARK 14. If $A$ is an uncountable set and $\left\{X_{a}: a \in A\right\}$ is a family of topological spaces such that $X=\Pi\left\{X_{a}: a \in A\right\}$ fails to be pseudocompact, then for some countably infinite subset $B$ of $A$, $\Pi\left\{X_{b}: b \in B\right\}$ must fail to be pseudocompact. Thus, by Theorem 11, if a product $X=\Pi\left\{X_{a}: a \in A\right\}$ of strongly functionally Hausdorff SW spaces fails to be an SW space, and if $A$ is uncountable, then for some countably infinite subset $B$ of $A, \Pi\left\{X_{b}: b \in B\right\}$ must also fail to be an SW space. Example 13 shows that the previous statement has no finite-countable analogue.

Before obtaining our main product theorem, we consider briefly a family of spaces which contains several useful examples.

It will be convenient below to call a space $X$ : completely regular at a point $x$ if for each neighborhood $V$ of $x$ in $X$ there exists a function $f \in C(X)$ which vanishes at $x$ and equals 1 on $X \backslash V$; and e-completely regular if there is a dense subset $D$ of $X$ such that $X$ is completely regular at each point of $D$.

While many examples of SW spaces are strongly functionally Hausdorff, several well known spaces which are SW spaces fail to be strongly functionally Hausdorff. For instance, Tychonoff's regular-closed but not completely regular space $T$ is an SW space [18], but one can show that if $p$ is the point at which $T$ fails to be completely regular, then the definition of strongly functionally Hausdorff is not satisfied by $p$ and neighborhoods of $p$; however, $T$ is certainly $e$-completely regular-its isolated points are dense. Likewise, the same is true of: S. H. Hechler's noncompact example [11] of a first countable regular-closed space which can be shown to be an SW space; and an example due to $H$. Herrlich [12] of a regular SW space which is not regular-closed. Theorem 15 shows that for such spaces Question 6(i) has an affirmative answer.

LEMMA 14. Let $X$ be a pseudocompact space which is e-complete$l y$ regular. Then $X$ is feebly compact. 
Proof. If $\mathscr{C}=\left\{U_{n}: n \in N\right\}$ is a 1-1 listing of a locally finite family of nonempty open sets, find continuous mappings $f_{n}: X \rightarrow[0,1]$ so that $f_{n}$ vanishes on the complement of $U_{n}$ and equals 1 at some point in $U_{n}, n \in N$, and note that $f=\sum n f_{n} \in C(X) / C^{*}(X)$. (By the local finiteness of $\mathscr{C}$, for each point $x \in X$ there is an open neighborhood $V$ of $x$ on which $f$ equals a mapping in $C(X)$, namely, $f_{1}+\cdots+k f_{k}$, for some $k \in N$.)

THEOREM 15. A product of e-completely regular SW spaces is an SW space if and only if it is pseudocompact.

Proof. The property $e$-completely regular is productive, and thus the result follows from Lemma 14 and Theorem 8.

Since every product of first countable feebly compact spaces is feebly compact [15] (see also [10]), Question 4 can be answered for $e$-completely regular spaces.

THEOREM 16. Every product of first countable e-completely regular SW spaces is an SW space.

One can easily obtain some extensions of results in [21] and [17] to e-completely regular spaces.

TheOREM 17. Let $X$ and $Y$ be pseudocompact spaces.

(i) If $X$ and $Y$ are e-completely regular spaces, then $X \times Y$ is pseudocompact if and only if $p_{X}$ is z-closed.

(ii) If $X$ is a k-space and $Y$ is an e-completely regular space, then $X \times Y$ is pseudocompact.

THEOREM 18. Every product of first countable e-completely regular pseudocompact spaces is pseudocompact.

REMARK 19. In Example 21 a simple example will be given of a functionally regular SW space which fails to be e-completely regular.

Let us return now to functionally regular spaces. Some lemmas will be obtained, and then several positive results will be given concerning products of functionally regular SW or pseudocompact spaces. We will conclude by generalizing Theorem 5 and answering Question 4 affirmatively for strongly functionally Hausdorff spaces.

Notation. Given a functionally Hausdorff space $X$, the closure in $X$ of a set $V$ will continue to be denoted by $\bar{V}$, and the closure in $w X$ of $V$ will be denoted by $\mathrm{Cl}_{w X} V$; the interior in $w X$ of $V$ 
will be denoted by $\operatorname{Int}_{w X} V$.

LeMma 20. Let $X$ be a pseudocompact functionally Hausdorff space, and suppose that $V$ is an open subset of the space $w X$. Then $\bar{V}$ is pseudocompact.

Proof. Suppose, on the contrary, that there exists a mapping $f \in C(\bar{V})$ with $\sup f(\bar{V})=\infty$. Since $V$ is dense in $\bar{V}$, there then exists a sequence $\left\{x_{n}\right\}$ in $V$ such that $\left\{f\left(x_{n}\right)\right\}$ is an increasing unbounded sequence of real numbers. Since the topology on the completely regular space $w(\bar{V})$ is finer than (or equal with) the subspace topology induced on the set $w(\bar{V})$ by $w X$, the set $V$ is an open subset of the space $w(\bar{V})$. Thus, for each $n \in N$, there is an open subset $V_{n}$ of $w(\bar{V})$ such that $x_{n} \in V_{n} \subset V$ and $f\left(V_{n}\right) \subset\left(f\left(x_{n-1}\right), f\left(x_{n+1}\right)\right)$. Let $\mathscr{V}=\left\{V_{n}: n \in N\right\}$.

Clearly, because $f \in C(\bar{V})$, $\mathscr{V}$ must be locally finite in $\bar{V}$. Since $\bar{V}$ is a closed subset of $X, \mathscr{V}$ is also locally finite in $X$.

By the complete regularity of $w(\bar{V})$, choose, for each $n \in N$, a mapping $f_{n} \in C(w(\bar{V}))$ such that $f_{n}$ vanishes on $\bar{V} \backslash V_{n}$ and equals 1 at some point of $V_{n}$. Since each $V_{n}$ is open in $X$ and $f_{n}(\bar{V} \backslash V)=0$, one may extend $f_{n}$ to a function $g_{n} \in C(X)$ by defining

$$
g_{n}(x)= \begin{cases}f_{n}(x) & \text { if } x \in \bar{V} \\ 0 & \text { if } x \in X \backslash \bar{V}\end{cases}
$$

By the local finiteness of $\mathscr{Y}$ in the space $X, \sum n g_{n} \in C(X) \backslash C^{*}(X)$, in contradiction of the assumption that $X$ is pseudocompact.

Although every open subspace of a pseudocompact completely regular space is known to have pseudocompact closure, Example 21 shows that a similar result does not hold, in general, even for functionally regular SW spaces.

EXAMPLe 21. The type of construction given here is due to H. Herrlich.

Let $H$ be a compact ordered space having greatest element $m$ and pairwise disjoint, dense subsets $E_{1}, E_{2}$, and $E_{3}$ such that $m \in E_{3}$ and $H=E_{1} \cup E_{2} \cup E_{3}$. Denote by $I=I(H)$ the space whose points are those of $H$ and whose topology has as a subbase $\mathscr{J} \cup\left\{E_{2}, E_{3}\right\}$, where $\mathscr{J}=$ the order topology on $H$. Then one can easily show that $w I=H$, so $I$ is an $\mathrm{SW}$ space.

If one takes $H=[0,1]$, then $I$ is a second countable, functionally regular SW space, but (as noted in [17]) if $a \in E_{3}$ and $V=E_{2}$, then $V$ is an open subset of $I$ and $\bar{V}$ is not pseudocompact, since $f(x)=$ $1 /(x-a)$ defines a function in $C(\bar{V}) \backslash C^{*}(\bar{V})$. 
LEMMA 22. Let $X$ be a pseudocompact functionally regular space, and suppose that $V$ is an open subset of $w X$. Then $\bar{V}=$ $\mathrm{Cl}_{w X} V$.

Proof. Since each open subset of $w X$ is open in $X, \bar{V} \subset \mathrm{Cl}_{w X} V$. To see that $\mathrm{Cl}_{w X} V \subset \bar{V}$, consider any point $x \in X \backslash \bar{V}$. By the functional regularity of $X$, there exists $f \in C(X)$ with $f(x) \notin f(\bar{V})$. By Lemma 20, $\bar{V}$ is pseudocompact. Thus, $f(\bar{V})$ is compact, and so using $f$, one can find a mapping $h \in C(X)$ which vanishes at $x$ and equals 1 on $\bar{V}$. Therefore, $x \notin \mathrm{Cl}_{w X} V$.

REMARK 23. In Lemma 22, functionally regular cannot be weakened to strongly functionally Hausdorff. In the strongly functionally Hausdorff SW space $X_{n}$ in Example 13 let $V=N \times\{1\}$. Then $V$ is open in $w X_{n}$, but $\left(\beta N \backslash G_{n}\right) \times\{2\} \subset\left(\mathrm{Cl}_{w X} V\right) \backslash \bar{V}$.

As in [18], a functionally Hausdorff space $X$ will be called weakly absolutely closed provided that every open filter base on $w X$ has an adherent point in $X$. It is easily seen that every weakly absolutely closed space is an SW space. In [18] the following theorem was obtained.

THEOREM 24 [18]. Let $X$ be a weakly absolutely closed space. If $Y$ is any SW space, then $X \times Y$ is an SW space.

We will see that the concept, weakly absolutely closed, is also quite helpful in the consideration of products of functionally regular SW spaces.

LEMMA 25. Let $X$ be a topological space. The following are equivalent.

(i) $X$ is weakly absolutely closed.

(ii) $X$ is an SW space, and for every open subset $V$ of $w X$, $\bar{V}=\mathrm{Cl}_{w X} V$.

Proof. (i) implies (ii). We will prove that $\mathrm{Cl}_{w X} V \subset \bar{V}$. Consider any point $p \in \mathrm{Cl}_{w X} V$. Let $\mathscr{C}$ be an open neighborhood base for $p$ in $w X$, and let $\mathscr{V}$ be the restriction of $\mathscr{C}$ to $V$. Then $\mathscr{V}$ is an open filter base on $w X$ and hence must have an adherent point $q$ in $X$. Since $q$ is also an adherent point of $\mathscr{V}$ in $w X, q$ must be $p$. Thus $p \in \bar{V}$.

(ii) implies (i). Let $\mathscr{W}$ be an open filter base on $w X$. Since $w X$ is compact, $\mathscr{W}$ has an adherent point in $w X$, but by the second statement in (ii), any adherent point of $\mathscr{W}$ in $w X$ is also an adherent point of $\mathscr{W}$ in $X$. 
EXAMPLE 26. In Example 21, let $H$ denote the one-point compactification of Alexandroff's long line, and let $m$ be the added point. Then for any zero set $Z$ with $m \in Z$, and for any neighborhood $V$ of $m$ in $I$ with $V \subset E_{3}$, one has $\phi \neq Z \cap\left(I \backslash\left(E_{1} \cup E_{3}\right)\right) \subset Z \cap(I \backslash \bar{V})$; for, any function in $C(H)=C(I)$ is eventually constant. Note, however, that $I$ satisfies Lemma 25 (ii).

COROLLARY 27. Every functionally regular SW space is weakly absolutely closed, but not every weakly absolutely closed space is strongly functionally Hausdorff.

LEMMA 28. Let $X$ be a functionally Hausdorff space. The following are equivalent.

(i) For each open subset $V$ of $w X, \bar{V}=\mathrm{Cl}_{w X} V$.

(ii) For each open subset $B$ of $X, B \subset \operatorname{Int}_{w X}\left(\mathrm{Cl}_{w X} B\right)$.

Proof. (i) implies (ii). Let $W=\mathrm{Cl}_{w X} B$ and $V=w X \backslash W$. By (i), $\bar{V}=\mathrm{Cl}_{w X} V$. Now consider any point $x \in B$. Since $B$ and $V$ are disjoint open subsets of $X$, one has $x \notin \bar{V}=\mathrm{Cl}_{w X} V$. Thus, $x$ has a neighborhood in $w X$ which misses $V$, i.e., $x \in \operatorname{Int}_{w X}(w X \backslash V)=$ $\operatorname{Int}_{w X}\left(\mathrm{Cl}_{w X} B\right)$.

(ii) implies (i). Suppose that $V$ is an open subset of $w X$. We will prove that $\bar{V} \supset \mathrm{Cl}_{w X} V$.

Since $X \backslash \bar{V}$ is an open subset of $X$, it follows from (ii) that

$$
X \backslash \bar{V} \subset \operatorname{Int}_{w X}\left(\mathrm{Cl}_{w X}(X \backslash \bar{V})\right)=\operatorname{Int}_{w X}\left(X \backslash \operatorname{Int}_{w X}(\bar{V})\right)=X \backslash \mathrm{Cl}_{w X}\left(\operatorname{Int}_{w X}(\bar{V})\right) .
$$

Hence $\left.\bar{V} \supset \mathrm{Cl}_{w X}\left(\operatorname{Int}_{w X} \bar{V}\right)\right)$, and since $\mathrm{V}$ is open in $w X$, we also have $\mathrm{Cl}_{w X}\left(\operatorname{Int}_{w X}(\bar{V})\right) \supset \mathrm{Cl}_{w X} V$. Therefore, $\bar{V} \supset \mathrm{Cl}_{w X} V$.

The next result gives the primary reason for our interest in the conditions in Lemma 28.

Lemma 29. Let $X=\Pi\left\{X_{a}: a \in A\right\}$, where each $X_{a}$ is a functionally Hausdorff space such that for every open set $B_{a}$ of $X_{a}$, one has $B_{a} \subset \operatorname{Int}_{w X_{a}}\left(\mathrm{Cl}_{w X_{a}} B_{a}\right)$. Then $w X=\Pi\left\{w X_{a}: a \in A\right\}$.

Proof. For an open subset $B_{a}$ of a factor $X_{a}$, Int ${ }_{w X_{a}}\left(\mathrm{Cl}_{w X_{a}} B_{a}\right)$ will be denoted $* B_{a}$. Let $T$ denote $\Pi\left\{w X_{a}: a \in A\right\}$.

In order to establish Lemma 29 , it will be enough to prove that $C(X) \subset C(T)$, for $T=w X$ if and only if $C(X)=C(T)$. Consider any function $f \in C(X)$.

Let $x=\left\{x_{a}\right\} \in X$, and let $P_{1}$ and $P$ be open subsets of the real line such that $\bar{P}_{1} \subset P$ and $f(x) \in P_{1}$. There exist a finite set $F=$ $\left\{a_{1}, \cdots, a_{n}\right\} \subset A$ and a basic open subset $B$ of $X, B=\cap\left\{p r_{a}^{-1}\left(B_{a}\right)\right.$ : 
$a \in F\}$, such that $x \in B$ and $f(B) \subset P_{1}$. We will prove that $f$ is continuous at $x$ with respect to the space $T$ by proving that $x$ also has a basic open neighborhood $B^{\prime}$ in $T$ such that $f\left(B^{\prime}\right) \subset P$, namely, the set $B^{\prime}=\cap\left\{p r_{a}^{-1}\left({ }^{*} B_{a}\right): a \in F\right\}$.

Select open sets $P_{i}, i=2, \cdots, n$, such that $\bar{P}_{i} \subset P_{i+1}, i=1, \cdots$, $n-1$, and $\bar{P}_{n} \subset P$. Then one can prove that for each $i, i=$ $1, \cdots, n$,

$(\#)_{i}: \quad f\left(\left(\cap\left\{p r_{a_{j}}^{-1}\left({ }^{*} B_{a_{j}}\right): j \leqq i\right\}\right) \cap\left(\cap\left\{p r_{a_{j}}^{-1}\left(B_{a_{j}}\right): i<j \leqq n\right\}\right)\right) \subset \bar{P}_{i}$.

For, suppose that $i$ is a positive integer $\leqq n$ and $(\sharp)_{k}$ is true for each positive integer $k<i$. Take and fix an arbitrary point $p=\left\{p_{a}\right\}$ in $\Pi\left\{X_{a}: a \neq a_{i}\right\}$ such that for each positive integer $j<i$, $p_{a_{j}} \in{ }^{*} B_{a_{j}}$, and for any $i<j \leqq n, p_{a_{j}} \in B_{a_{j}}$. The function $g$ determined by the rule $g(t)=f\left(t^{\prime}\right)$, where

$$
t_{a}^{\prime}= \begin{cases}p_{a}, & \text { if } a \neq a_{i} \\ t, & \text { otherwise }\end{cases}
$$

is in $C\left(X_{a_{i}}\right)$. Furthermore, $g\left(B_{a_{i}}\right) \subset P_{i}$. Since $g \in C\left(X_{a_{i}}\right)=C\left(w X_{a_{i}}\right)$, one must have $g\left(* B_{a_{i}}\right) \subset g\left(\mathrm{Cl}_{w X} B_{a_{i}}\right) \subset \bar{P}_{i}$. Because the latter holds for any such point $p$ and corresponding function $g,(\#)_{i}$ is true.

By $(\sharp)_{n}$, we have $f\left(B^{\prime}\right) \subset \bar{P}_{n} \subset P$. Also, $x \in B^{\prime}$, for $x \in B$, and, by hypothesis, $B \subset B^{\prime}$. So, $B^{\prime}$ is a neighborhood of $x$ in $T$, and $f\left(B^{\prime}\right) \subset P$, which shows that $f$ is continuous at $x$ with respect to $T$. Thus $f \in C(T)$.

Our main theorem can now be given.

THEOREM 30. Every product of weakly absolutely closed spaces is weakly absolutely closed.

Proof. Let $X=\Pi\left\{X_{a}: a \in A\right\}$, where each $X_{a}$ is weakly absolutely closed. It is enough to prove that condition (ii) of Lemma 25 is satisfied.

By Theorem 3 and Lemmas 25, 28, and 29, $X$ is an SW space and $w X=\Pi\left\{w X_{a}: a \in A\right\}$.

Consider any open subset $V$ of $w X$. We wish to prove that $\bar{V} \supset \mathrm{Cl}_{w X} V$. Let $x$ be an arbitrary point of $X \backslash \bar{V}$. There exists a basic open neighborhood of $x$ in $X$, say

$$
B=\cap\left\{p r_{f}^{-1}\left(B_{f}\right): f \in F\right\},
$$

where $F$ is finite and each $B_{f}, f \in F$, is an open subset of $X_{f}$, such that $V \cap B=\phi$. Because $V$ is an open subset of $w X$, it follows from the latter that $V \cap \mathrm{Cl}_{w X} B=\phi$. Thus $V \cap \operatorname{Int}_{w X}\left(\mathrm{Cl}_{w X} B\right)=\dot{\phi}$. Since $w X=\prod\left\{w X_{a}: a \in A\right\}$, we have 


$$
\operatorname{Int}_{w X}\left(\mathrm{Cl}_{w X} B\right)=\cap\left\{p r_{f}^{-1}\left(\operatorname{Int}_{w X_{f}}\left(\mathrm{Cl}_{w X_{f}}\left(B_{f}\right)\right)\right): f \in F\right\},
$$

which contains $B$ (each $X_{f}$ satisfies Lemma 28 (ii)). Therefore, $x \notin$ $\mathrm{Cl}_{w X} V$, for there exists an open subset of $w X$ which contains $x$ and misses $V$, namely, the set $\operatorname{Int}_{w X}\left(\mathrm{Cl}_{w X} B\right)$.

COROLLARY 31. Every product of functionally regular SW spaces is an $\mathrm{SW}$ space.

COROLLARY 32. Every product of SW spaces in which points are zero sets is an $\mathrm{SW}$ space.

REMARK 33. In a strongly functionally Hausdorff first countable space $X$ each point is a zero set, for if $\left\{V_{n}: n \in N\right\}$ is a neighborhood base at $x$, and if $f_{n}: X \rightarrow[0,1]$ is chosen from $C(X)$ with $f_{n}(x)=$ $0 \notin f_{n}\left(X \backslash \bar{V}_{n}\right)$, then $f=\sum 2^{-n} f_{n} \in C(X)$ and $f^{-1}(0)=\{x\}$. Likewise, in a weakly absolutely closed first countable space $X$ each point must be a zero set, for if $\mathscr{U}$ is a neighborhood base in $X$ for a point $x$, then $\left\{\operatorname{Int}_{w X}\left(\mathrm{Cl}_{w X} V\right): V \in \mathscr{Q}\right\}$ is a neighborhood base in $w X$ for $x$. Thus, Corollary 32 answers Question 4 for products of weakly absolutely closed and strongly functionally Hausdorff spaces.

We will give an example below which shows that there exists a first countable SW space containing a point that is not a zero set. First a lemma is needed.

LEMMA 34. Every weakly absolutely closed, e-completely regular space $X$ is Hausdorff-closed.

Proof. Let $\mathscr{Q}$ be an open filter base on $X$. For each set $U \in \mathscr{C}$, it follows from the $e$-complete regularity of $X$ that the open subset $\operatorname{Int}_{w X} U$ of $w X$ has the property that, with respect to the space $X$, it is a dense open subset of $U$. Thus $\mathscr{W}=\left\{\operatorname{Int}_{w X} U\right.$ : $U \in \mathscr{Q}\}$ is an open filter base on $w X$, and, in the space $X$, $\mathscr{C}$ and $\mathscr{W}$ have the same adherent points. Since $X$ is weakly absolutely closed, $\mathscr{W}$ and, hence, $\mathscr{C}$ must have an adherent point in the space $X$.

Although it is well known that every regular, Hausdorff-closed space is compact, I do not know the answer to the following.

Question 35. Is every regular, weakly absolutely closed space compact?

EXAMPLE 36. Let $X$ be the noncompact, separable, first countable regular space $(S, \mathscr{S})$ in [11] which Hechler proved is a regular- 
closed space. Each point $x \in X \backslash\{a\}$ has a neighborhood base consisting of compact clopen sets. Let $a X$ be the one-point compactification of $X \backslash\{a\}$, where $a X$ has the same points as those of $X$. Then every open subset of the compact space $a X$ is open in $X$, so $X$ is functionally Hausdorff, as well as regular-closed, and hence is an SW space ([4], [6]). Since its isolated points form a dense subset, $X$ is $e$-completely regular. Therefore, by Lemma 34, $\{a\}$ cannot be a zero set, for if it were then every point of $X$ would be a zero set, and $X$ itself would be functionally regular and thus compact.

Some other product theorems which can be obtained are these.

THEOREM 37. Let $X$ be a weakly absolutely closed space. If $Y$ is any pseudocompact space, then so is $X \times Y$.

Proof. This theorem is a consequence of $[17,4.10]$ and can also be obtained directly from a short argument (similar to the proof of Lemma 29) which shows that $C(X \times Y)=C(w X \times Y)$.

THEOREm 38. Let $X=\Pi\left\{X_{a}: a \in A\right\}$, where each $X_{a}$ is a pseudocompact functionally regular space. Then $w X=\Pi\left\{w X_{a}: a \in A\right\}$.

CoROllary 39. Let $X=\Pi\left\{X_{a}: a \in A\right\}$, where each $X_{a}$ is a pseudocompact space in which points are zero sets. Then $X$ is pseudocompact.

Proof. A $G_{\delta}$-point in a pseudocompact completely regular space has a countable neighborhood base [10], so each $w X_{a}$ is first countable, and $\Pi\left\{w X_{a}: a \in A\right\}$ is pseudocompact.

REMARK 40. Some of the results above might lead one to conclude that weakly absolutely closed spaces must be very close to being compact. On the contrary, an example in $[17,3.5]$ is a first countable space $X$ such that $w X=[0,1]$, but which contains nonempty open subsets $\left\{U_{n}: n \in N\right\}$ such that each $U_{n} \supset \bar{U}_{n+1}$ and $\cap\left\{U_{n}: n \in N\right\}=\phi$.

On the other hand, as C. E. Aull and the referee have pointed out, if an SW space $Y$ is so close to being compact that it is Hausdorff-closed, then $Y$ must also be a strongly functionally Hausdorff space. To verify their result, recall that $s Y$, the semiregularization of a Hausdorff-closed, functionally Hausdorff space $Y$, is compact (e.g., see [6]), and hence for any open neighborhood $V$ in $Y$ of a point $y$, the set $W=(\bar{V})^{0}$ is open in $s Y=w Y$ and has the property that $\bar{W}=\bar{V}$; thus, there exists $f \in C(w Y)$ which vanishes at $y$ and equals 1 on $Y \backslash W$, and so one has $f(y) \notin f(Y \backslash \bar{V})$. 
In [18, Example 10] an example was given of a countably compact, Hausdorff-closed SW space $Y$ which fails to be a $k$-space. Since every function in $C(Y)$ is eventually constant, the space $Y$ can also be used to show that although every Hausdorff-closed SW space must be strongly functionally Hausdorff, such a space may fail to be functionally regular- $Y$ fails to be functionally regular at the first uncountable ordinal.

\section{REFERENCES}

1. C. E. Aull, Functionally regular spaces, Indag. Math., (1976), Proc. Kon. Ned. Akad. Wetensch., A79 (1976), 281-288.

2. - Approximation of continuous functions on pseudocompact spaces, Proc. Amer. Math. Soc., 81 (1981), 490-494.

3. R. W. Bagley, E. H. Connell, and J. D. MoKnight, Jr., On properties characterizing pseudo-compact spaces, Proc. Amer. Math. Soc., 9 (1958), 500-506.

4. B. Banaschewski, On the Weierstrass-Stone approximation theorem, Fund. Math., 44 (1957), 249-252.

5. — Extensions of topological spaces, Canad. Math. Bull., 7 (1964), 1-22.

6. M. P. Berri, J. R. Porter, and R. M. Stephenson, Jr., A survey of minimal topological spaces, General Topology and its Relations to Modern Analysis and Algebra, III (Proc. Conf., Kanpur, 1968). pp. 93-114. Academia, Prague, 1971.

7. W. W. Comfort, A nonpseudocompact product space whose finite subproducts are pseudocompact, Math. Ann., 170 (1967), 41-44.

8. W. W. Comfort and A. W. Hager, The projection mapping and other continuous functions on a product space, Math. Scand., 28 (1971), 77-90.

9. A. J. d'Aristotle, On the extension of mappings in Stone-Weierstrass spaces, Trans. Amer. Math. Soc., 208 (1975), 91-101.

10. I. Glicksberg, Stone-Čech compactifications of products, Trans. Amer. Math. Soc., 90 (1959), 369-382.

11. S. H. Hechler, Two R-closed spaces revisited, Proc. Amer. Math. Soc., 56 (1976), 303-309.

12. H. Herrlich, Regular-closed, Urysohn-closed, and completely Hausdorff-closed spaces, Proc. Amer. Math. Soc., 26 (1970), 695-698.

13. E. Hewitt, Certain generalizations of the Weierstrass approximation theorem, Duke Math. J., 14 (1947), 419-427.

14. T. Isiwata, Mappings and spaces, Pacific J. Math., 20 (1967), 455-480.

15. C. T. Scarborough and A. H. Stone, Products of nearly compact spaces, Trans. Amer. Math. Soc., 124 (1966), 131-147.

16. R. M. Stephenson, Jr., Spaces for which the Stone-Weierstrass theorem holds. Trans. Amer. Math. Soc., 133 (1968), 537-546.

17. - Pseudocompact spaces, Trans. Amer. Math. Soc., 134 (1968), 437-448.

18. - Product spaces for which the Stone-Weierstrass theorem holds, Proc. Amer. Math. Soc., 21 (1969), 284-288.

19. - Products of nearly compact spaces, Proceedings of the University of Oklahoma Topology Conference Dedicated to Robert Lee Moore (1972), 310-320. Univ. of Oklahoma, Norman, Oklahoma, 1972.

20. - Product spaces and the Stone-Weierstrass theorem, General Topology and its Applications, 3 (1973), 77-79.

21. H. Tamano, A note on the pseudocompactness of the product of two spaces, Mem. Coll. Sci., Univ. Kyoto Ser. A Math., 33 (1960/61), 225-230.

22. W. T. van Est and H. Freudenthal, Trennung durch stetige Funktionen in topo- 
logischen Raumen, Indag. Math., 15 (1961), 359-368.

23. S. Willard, General Topology, Addison-Wesley, Reading, Mass., 1970.

Received March 21, 1980 and in revised form May 26, 1980. This research was partially supported by two University of South Carolina grants administered through the Research and Productive Scholarship Program and through the College of Science and Mathematics.

University of South Carolina

CoLUMBiA, SC 29208 


\section{PACIFIC JOURNAL OF MATHEMATICS}

\section{EDITORS}

DONALD BABBITT (Managing Editor)

University of California

Los Angeles, California 90024

\section{Hugo Rossi}

University of Utah

Salt Lake City, UT 84112

C. C. MOore and Arthur AGuS

University of California

Berkeley, CA 94720
J. DugundJI

Department of Mathematics University of Southern California Los Angeles, California 90007

R. FinN and J. Milgram Stanford University Stanford, California 94305

ASSOCIATE EDITORS
R. ARENS
E. F. BECKENBACH
B. H. NeumanN
F. WOLF
K. YOSHIDA

\section{SUPPORTING INSTITUTIONS}

UNIVERSITY OF ARIZONA

UNIVERSITY OF BRITISH COLUMBIA

CALIFORNIA INSTITUTE OF TECHNOLOGY

UNIVERSITY OF CALIFORNIA

MONTANA STATE UNIVERSITY

UNIVERSITY OF NEVADA, RENO

NEW MEXICO STATE UNIVERSITY

OREGON STATE UNIVERSITY
UNIVERSITY OF OREGON

UNIVERSITY OF SOUTHERN CALIFORNIA

STANFORD UNIVERSITY

UNIVERSITY OF HAWAII

UNIVERSITY OF TOKYO

UNIVERSITY OF UTAH

WASHINGTON STATE UNIVERSITY

UNIVERSITY OF WASHINGTON 


\section{Pacific Journal of Mathematics}

Vol. 99, No. $1 \quad$ May, 1982

Mariano Giaquinta, Jindrich Necas, O. John and J. Stará, On the

regularity up to the boundary for second order nonlinear elliptic systems . . 1

Siegfried Graf, Realizing automorphisms of quotients of product $\sigma$-fields . . 19

Alfred Washington Hales and Ernst Gabor Straus, Projective colorings . . . 31

Sandra Hayes, The weak Nullstellensatz for finite-dimensional complex

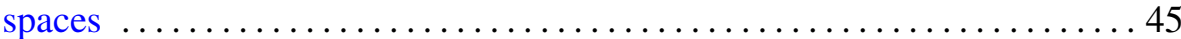

Gerald Norman Hile and Murray Harold Protter, The Cauchy problem

and asymptotic decay for solutions of differential inequalities in Hilbert

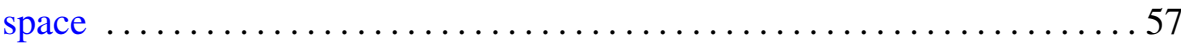

Robert D. Little, Projective space as a branched covering with orientable

branch set ......................................... 89

Jaroslav Mach, On the proximinality of Stone-Weierstrass subspaces . . . . . 997

John C. Morgan, II, On product bases ...................... 105

K. Balakrishna Reddy and P. V. Subrahmanyam, Altman's contractors

and fixed points of multivalued mappings . .................. 127

James Ted Rogers Jr., Decompositions of homogeneous continua . . . . . . . 137

Ahmed Ramzy Sourour, Characterization and order properties of

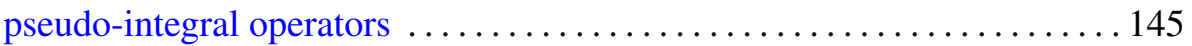

Robert Moffatt Stephenson Jr., Pseudocompact and Stone-Weierstrass

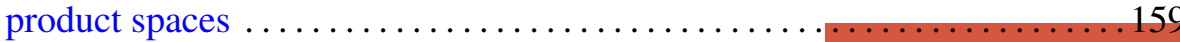

Bruce Stewart Trace, On attaching 3-handles to a 1-connected

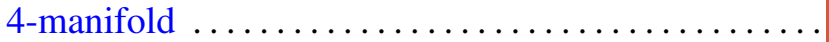

Akihito Uchiyama, The construction of certain BMO functions and the corona problem

Thomas Alva Whitehurst, An application of orthogonal polynomials to random walks ..............................

David J. Winter, Root locologies and idempotents of Lie and nonassociative algebras

William Robin Zame, The classification of uniform algebras on plane domains 